\title{
Controles alternados das ordenhas da manhã e da tarde para estimação da produção de leite na lactação
}

[Morning and afternoon tests for estimating lactation milk yield]

\author{
N.M. Teixeira ${ }^{1,4}$, A.F. Freitas ${ }^{1,4}$, C.N. Costa ${ }^{1,4}$, J.A. Cobuci ${ }^{2,4}$, R.B. Barra ${ }^{3}$ \\ ${ }^{1}$ Embrapa Gado de Leite \\ Rua Eugênio do Nascimento, 610 \\ 36038-330 - Juiz de Fora, MG \\ ${ }^{2}$ Faculdade de Agronomia - UFRGS \\ ${ }^{3}$ ACGHMG - Juiz de Fora, MG \\ ${ }^{4}$ Bolsista do $\mathrm{CNPq}$
}

\begin{abstract}
RESUMO
Determinaram-se procedimentos para estimar produção diária de leite usando controles da manhã (AM) ou da tarde (PM) e comparar sistemas de controle para previsão da produção de leite em 305 dias usando várias combinações de controles AM e PM. Utilizaram-se 20.328 produções do dia de controle de 1.516 lactações de vacas ordenhadas duas vezes ao dia, em 96 rebanhos supervisionados pela Associação dos Criadores de Gado Holandês de Minas Gerais e registradas no período de 2000 a 2003. Sete procedimentos foram usados para estimação da produção diária. Correlações entre produções observada e estimada, médias e desvios-padrão das diferenças entre elas e desvios-padrão das produções estimadas foram os critérios para comparação de procedimentos. As estimativas obtidas com a ordenha da manhã foram mais acuradas do que as obtidas com as da ordenha da tarde. O procedimento em que se incluiu uma regressão para cada classe de intervalo de ordenhas e uma regressão sobre dias em lactação foi o selecionado para estimação da produção diária. Quatro sistemas para previsão da produção de leite em 305 dias foram comparados. Os sistemas com alternância mensal dos controles AM-PM apresentaram melhores resultados do que aqueles em que se utilizaram somente o controle AM ou somente PM. As correlações entre produção estimada e observada foram 0,99 e 0,97 , respectivamente, para os primeiros e os últimos sistemas. Alternância dos controles AM-PM, iniciando-se com o controle AM, foi o sistema mais acurado de estimação.
\end{abstract}

Palavras-chave: gado de leite, controle leiteiro, estádio da lactação, estimação da produção, intervalo de ordenhas

\begin{abstract}
Procedures to estimate daily yield from either morning (AM) or evening (PM) test weighings and to compare different systems of testing for accuracy of predicting 305-day milk yield using various combinations of AM and PM production records were determined. Data of 20,328 test-day records of 1,516 cows milked twice-a-day, from 96 Holstein Dairy Cattle Association herds in Minas Gerais State and recorded from 2000 to 2003. Seven procedures were used to estimate daily yields. Comparisons were made on the basis of correlations between true and estimated daily yields, average difference between the estimated and actual values and also the variances of differences. Morning weighings predicted daily yield more accurately than evening weighings. The procedure which included a regression for each milking interval class and a regression on days in milk were selected for estimation of daily yields. Four systems for predicting 305-day milk yield were compared. Alternating AM-PM testing systems on monthly frequency showed better results than testing always in the AM or always in the PM. Correlations between
\end{abstract}

Recebido em 29 de setembro de 2004

Aceito em 6 de setembro de 2005

E-mail: nilson@cnpgl.embrapa.br 
observed and estimated 305-day lactation yields were 0.99 and 0.97, respectively, for the first and later systems. Alternating AM-PM testing starting with an AM test was the most accurate system of estimation.

Keywords: dairy cattle, milking records, prediction, lactation stage, milking interval

\section{INTRODUÇ̃̃O}

No Brasil, a produção na lactação tem sido a base de informação sobre produção das vacas usadas em avaliações genéticas de vacas e touros (Costa et al., 2003; Verneque et al., 2003). Tradicionalmente, ela é calculada usando-se a produção diária de vacas individuais, controladas uma vez ao mês, requerendo para tanto visitas mensais de um controlador à fazenda. Em virtude, principalmente, dos custos crescentes do controle, poucos rebanhos são controlados (Costa et al., 2004). Para reverter tal situação, estratégias de controle diferentes da atual poderiam ser usadas. Variações de estratégias implicariam alterações da freqüência do controle se ele for realizado por controlador oficial ou pelo produtor. Estratégias alternativas que possibilitem custo o mais baixo possível por vaca requerem investigar a sua precisão para estimar a produção na lactação para condições brasileiras.

Uma alternativa para o controle mensal é o controle de uma única ordenha, em vez de duas, alternando-se as ordenhas da manhã e da tarde em meses subseqüentes. As vantagens potenciais deste procedimento comparado ao controle mensal seriam redução do tempo de permanência do controlador na fazenda, menor interferência na rotina da fazenda, menor custo para o criador e maior número de rebanhos em controle.

A idéia de se alternar os controles da manhã ou da tarde em vez de se usar a produção de um dia não é recente. Desde o final da década de 60, nos Estados Unidos, vêm sendo realizados estudos sobre este procedimento, os quais geralmente o recomendam como uma opção alternativa eficiente (Putman e Gilmore, 1968, 1969; McDaniel, 1969; Wiggans, 1981).

Putman e Gilmore (1968) compararam pesagens mensais (controle oficial), pesagens da manhã e tarde alternadas (controle AM-PM) e pesagens bimensais com a produção verdadeira calculada usando as produções diárias. Eles concluíram que as estimativas usando o controle AM-PM eram mais precisas do que as bimensais, mais aceitas pelos produtores, interferiam menos na rotina na fazenda, possibilitavam menor custo por vaca e, também, maior renda para o controlador. Por este procedimento, a produção de uma única ordenha da manhã ou da tarde era duplicada para estimar a produção do dia de controle.

O controle mensal com alternância das ordenhas da manhã e da tarde foi, também, superior aos sistemas com todos os controles da manhã, ou todos da tarde, bimensal diário e pouco menos preciso do que o controle da produção diária mensal para estimação da produção na lactação (Dickinson e McDaniel, 1970; Smith e Pearson, 1981).

Em alguns trabalhos, os autores encontraram efeito do intervalo das ordenhas da manhã e da tarde sobre a produção diária e sugeriram fatores para sua estimação com ajustamento para o intervalo delas (Everett e Wadel, 1970; Schaeffer e Rennie, 1976; Smith e Pearson,1981; Lee e Wardrop, 1984, Cassandro et al. 1995).

Outra fonte importante de variação que deve ser considerada ao se estimarem produções diárias é o estádio de lactação (Schaeffer e Rennie, 1976; Lee e Wardrop, 1984). Schaeffer e Rennie (1976) estimaram fatores para obtenção da produção diária usando a ordenha da manhã ou da tarde, considerando o intervalo de ordenhas e o mês da lactação. Os fatores obtidos foram mais precisos para estimação da produção diária do que simplesmente duplicando-se a produção da manhã ou da tarde. Ainda, as produções diárias estimadas por pesagens da manhã foram mais precisas do que as estimadas pela ordenha da tarde. Além disso, a alternância de ordenhas da manhã e da tarde produziu estimativas da produção aos 305 dias pouco menos precisas do que as obtidas usando-se as produções diárias. A ordem e a estação de parição podem também afetar as estimativas das produções (Schaeffer et al., 2000).

No Brasil, ainda não se dispõe de um sistema para estimação da produção na lactação, com 
número reduzido de controles, capaz de diminuir o custo do controle por vaca. Tais sistemas precisam ser estudados quanto a sua precisão para estimar a produção na lactação. Os objetivos deste trabalho foram: determinar um procedimento para estimação da produção diária com base em pesagens do leite da manhã ou da tarde e comparar sistemas para estimação da produção até 305 dias usando controles da manhã e/ou da tarde.

\section{MATERIAL E MÉTODOS}

Foram usados registros relativos a 20.328 produções de leite, em duas ordenhas do dia de controle, de 1.516 lactações de duas ordenhas, iniciadas no período de 2000 a 2003, em 96 rebanhos supervisionados pela Associação dos Criadores de Gado Holandês de Minas Gerais (ACGHMG). Os registros consistiram de produções da ordenha da manhã e da tarde, hora de início das ordenhas, estádio da lactação e idade da vaca ao parto.

O intervalo de ordenhas para a ordenha da manhã foi calculado como o tempo entre a ordenha da tarde anterior e o início da ordenha da manhã e, para a ordenha da tarde, pela diferença entre a ordenha da manhã e da tarde do mesmo dia. A produção diária foi obtida pela soma das produções da manhã e da tarde do dia de controle. Para possibilitar melhor qualidade dos dados disponíveis, os controles com produções menores do que $3,0 \mathrm{~kg}$ e maiores do que $40 \mathrm{~kg}$ foram descartados. $\mathrm{O}$ número mínimo de controles requerido por vaca em cada lactação foi oito.

As lactações foram separadas em primeira e outras, sendo consideradas primeiras quando o controle iniciava antes dos 40 meses de idade da vaca. Os intervalos das ordenhas, em minutos, foram separados em sete classes: $<540,540-$ $600,601-660,661-720,721-780,781-810$ e $>810$. Os estádios de lactação foram agrupados em classes de 1 a 12, calculados pelo número de dias em lactação dividido por 30 e acrescido de uma unidade.

Os procedimentos para estimação da produção diária $\left(\mathrm{y}_{\mathrm{d}}\right)$ com base em produções parciais $\left(\mathrm{y}_{\mathrm{p}}\right)$ de uma única ordenha aplicados aos mesmos conjuntos de dados foram: 1) fator $\mathrm{F}=$ média de $\mathrm{y}_{\mathrm{d}} /$ média $\mathrm{y}_{\mathrm{p}}$ (um fator foi estimado para cada classe de intervalo de ordenha e ordem de parto); 2) duplicação da produção parcial, $y_{d}=2 y_{p}$ (admite-se que a produção diária esperada seja duas vezes a produção da manhã ou da tarde); 3) regressão simples, $\mathrm{y}_{\mathrm{d}}=\mathrm{b}_{0}+\mathrm{b}_{1} \mathrm{y}_{\mathrm{p}}$ (a produção diária é regredida sobre a produção parcial da manhã ou da tarde - uma equação é usada para todo o conjunto de dados); 4) regressão simples + intervalo de ordenhas (IO) como co-variável, $\mathrm{y}_{\mathrm{d}}=\mathrm{b}_{0}+\mathrm{b}_{1} \mathrm{y}_{\mathrm{p}}+\mathrm{b}_{2}$ (IO) (mais um coeficiente de regressão foi adicionado, isto é, o efeito médio de IO, em minutos, sobre a produção diária foi também considerado); 5) regressão simples para cada classe de intervalo de ordenhas $i, y_{d}=b_{0}^{i}+$ $\mathrm{b}_{1}^{\mathrm{i}} \mathrm{y}_{\mathrm{p}}^{\mathrm{i}}$;6) regressão simples para cada classe de intervalo de ordenhas $\mathrm{i}+$ regressão sobre dias em lactação (DEL), $\mathrm{y}_{\mathrm{d}}=\mathrm{b}_{0}^{\mathrm{i}}+\mathrm{b}_{1}^{\mathrm{i}} \mathrm{y}_{\mathrm{p}}^{\mathrm{i}}+\mathrm{b}_{2}^{\mathrm{i}}(\mathrm{DEL}-$ 158) (para cada classe i de IO, usou-se a regressão simples e incluiu-se a regressão sobre DEL para ajustar para o efeito de estádio de lactação - como referência, considerou-se DEL igual a 158, que é o ponto médio da lactação); 7) regressão simples para cada combinação de ordem de parição (i), classe de intervalo de ordenha (j) e estádio da lactação $(k), y_{d}=b_{0}^{i j k}+$ $\mathrm{b}_{1}^{\mathrm{ijk}} \mathrm{y}_{\mathrm{p}}^{\mathrm{ijk}}$

Para comparação desses procedimentos, usaramse correlações entre produções observadas e estimadas, médias e desvios-padrão das diferenças entre elas e desvios-padrão das produções estimadas. O melhor procedimento foi usado para estimação da produção diária.

Compararam-se, em seguida, quatro sistemas de controle para obtenção da produção até 305 dias: 1) alternância dos controles da manhã e da tarde, iniciando-se com o controle da manhã, 2) alternância, iniciando-se com o controle da tarde, 3) somente os controles da manhã, e 4) somente os controles da tarde. Para estimação da produção até 305 dias, usou-se o método oficial brasileiro (Brasil, 1986).

\section{RESULTADOS E DISCUSSÃO}

Comparadas à primeira lactação, as outras lactações apresentaram maiores médias e 
variâncias da produção de leite. As médias para os controles da manhã e da tarde foram $11,79 \pm 3,22$ e $9,91 \pm 3,14$ e $13,60 \pm 4,32$ e $11,06 \pm 4,21$, respectivamente, para primeira e outras lactações (Tab. 1). Também as médias e as variâncias diferiram com os intervalos de ordenha. É importante que essas diferenças de variâncias sejam consideradas e que os efeitos de ordem de parição e intervalo de ordenhas sejam contemplados nos procedimentos para estimação das produções diárias. $\mathrm{Na}$ Tab. 2 encontram-se correlações entre produções diárias observadas e estimadas (r), desvios-padrão das produções diárias estimadas $(\sigma)$ e erros médio de estimação usando os diferentes procedimentos. Estes resultados são semelhantes aos de Schaeffer e Rennie (1976), Lee e Wardrop (1981) e Cassandro et al. (1995), isto é, as estimativas obtidas com as ordenhas da manhã foram mais acuradas do que as das ordenhas da tarde.

Tabela 1. Média e desvio-padrão (DP) da produção de leite das ordenhas da manhã e da tarde para primeira e outras lactações por intervalo de ordenha

\begin{tabular}{|c|c|c|c|c|c|c|c|c|}
\hline \multirow{3}{*}{$\begin{array}{l}\text { Intervalo } \\
\text { (minutos) }\end{array}$} & \multicolumn{4}{|c|}{ Manhã } & \multicolumn{4}{|c|}{ Tarde } \\
\hline & \multicolumn{2}{|c|}{ Primeira } & \multicolumn{2}{|c|}{ Outras } & \multicolumn{2}{|c|}{ Primeira } & \multicolumn{2}{|c|}{ Outras } \\
\hline & Média & DP & Média & $\mathrm{DP}$ & Média & DP & Média & DP \\
\hline$<540$ & & & & & 9.3 & 2.48 & 7.7 & 3.09 \\
\hline $540-600$ & & & & & 8.5 & 2.80 & 9.5 & 3.72 \\
\hline $601-660$ & 11.8 & 3.45 & 13.8 & 4.08 & 10.0 & 3.16 & 11.4 & 4.17 \\
\hline $661-720$ & 11.4 & 3.20 & 13.0 & 4.20 & 10.5 & 3.08 & 12.2 & 4.13 \\
\hline $721-780$ & 12.1 & 3.26 & 14,0 & 4.51 & 11.0 & 2.45 & 11.6 & 4.18 \\
\hline $781-810$ & 11.8 & 2.88 & 13.2 & 3.80 & & & & \\
\hline$>810$ & 11.9 & 3.22 & 13.2 & 4.30 & & & & \\
\hline $\mathrm{N}^{\mathrm{o}}$ registros & & & & & & & & \\
\hline Média & & & & & & & & \\
\hline DP & & & & & & & & \\
\hline $\mathrm{N}^{\mathrm{o}}$ registros & & & & & & & & \\
\hline Média & & & & & & & & \\
\hline DP & & & & & & & & \\
\hline
\end{tabular}

Tabela 2. Correlações (r) entre produções diárias observadas e estimadas, médias e desvios-padrão $(\mathrm{kg})$ das diferenças entre produções estimadas e observadas (erro médio) e desvios-padrão $(\sigma)$ das produções estimadas usando-se ordenhas da manhã ou da tarde em diferentes procedimentos de estimação da produção diária

\begin{tabular}{|c|c|c|c|c|}
\hline & Procedimento & $\mathrm{r}$ & Erro-médio & $\sigma$ \\
\hline \multirow{7}{*}{ Manhã } & 1 & 0,956 & $-0,1178 \pm 2,1565$ & 7,2766 \\
\hline & 2 & 0,941 & $-2,1013 \pm 2,6608$ & 7,8505 \\
\hline & 3 & 0,941 & $0,0000 \pm 2,4552$ & 6,8248 \\
\hline & 4 & 0,956 & $0,0001 \pm 2,1258$ & 6,9322 \\
\hline & 5 & 0,956 & $0,0000 \pm 2,1157$ & 6,9353 \\
\hline & 6 & 0,957 & $-0,0001 \pm 2,1060$ & 6,9060 \\
\hline & 7 & 0,958 & $-0,0290 \pm 2,0720$ & 6,9627 \\
\hline \multirow{7}{*}{ Tarde } & 1 & 0,931 & $-1,0977 \pm 3,4254$ & 8,9370 \\
\hline & 2 & 0,937 & $2,1013 \pm 2,6608$ & 7,5989 \\
\hline & 3 & 0,937 & $0,0000 \pm 2,5365$ & 6,7950 \\
\hline & 4 & 0,949 & $0,0000 \pm 2,2780$ & 6,8862 \\
\hline & 5 & 0,950 & $0,0000 \pm 2,2607$ & 6,8919 \\
\hline & 6 & 0,950 & $-0,0002 \pm 2,2502$ & 6,8641 \\
\hline & 7 & 0,953 & $0,0000 \pm 2,2001$ & 6,9117 \\
\hline
\end{tabular}

Arq. Bras. Med. Vet. Zootec., v.58, n.3, p.394-400, 2006
Duplicar as produções dos controles da manhã (procedimento 2) foi o que mais subestimou a produção diária, indicando que o intervalo das ordenhas e o mês de lactação precisam ser considerados.

Embora as diferenças sejam pequenas, as correlações tenderam a aumentar com a complexidade do procedimento, exceto para o procedimento 7, para o qual a correlação foi baixa para a ordenha da manhã. As correlações entre produções das ordenhas da tarde foram ligeiramente inferiores às das produções correspondentes das ordenhas da manhã.

Com exceção dos procedimentos 1, 2 e 7 para ordenha da manhã, todos os demais apresentaram desvios-padrão das produções diárias estimadas menores do que desvio-padrão das produções diárias observadas, de $6,970 \mathrm{~kg}$.

Nas Fig. 1 e 2 representam-se as médias dos erros de estimação (observado-estimado) da 
produção diária usando produções da manhã e da tarde, respectivamente. O procedimento 6 , que consistiu de 10 fórmulas (Tab. 3), foi o escolhido para a estimação da produção diária, embora, pelo procedimento 7 , o erro médio em cada estádio de lactação tendesse a zero. Nesse utilizou-se uma regressão para cada estádio de lactação, num total de 120 fórmulas diferentes (duas ordens de parição $\mathrm{x}$ cinco classes de intervalo de ordenha x 12 estádios de lactação). Para os outros procedimentos, entretanto, a produção diária foi subestimada no início da lactação e superestimada no final (Fig. 1 e 2). Exceto para lactações curtas, esse padrão dos erros de estimação não tem efeito significativo sobre a produção estimada de 305 dias, uma vez que erros positivos e negativos tendem a se cancelar.
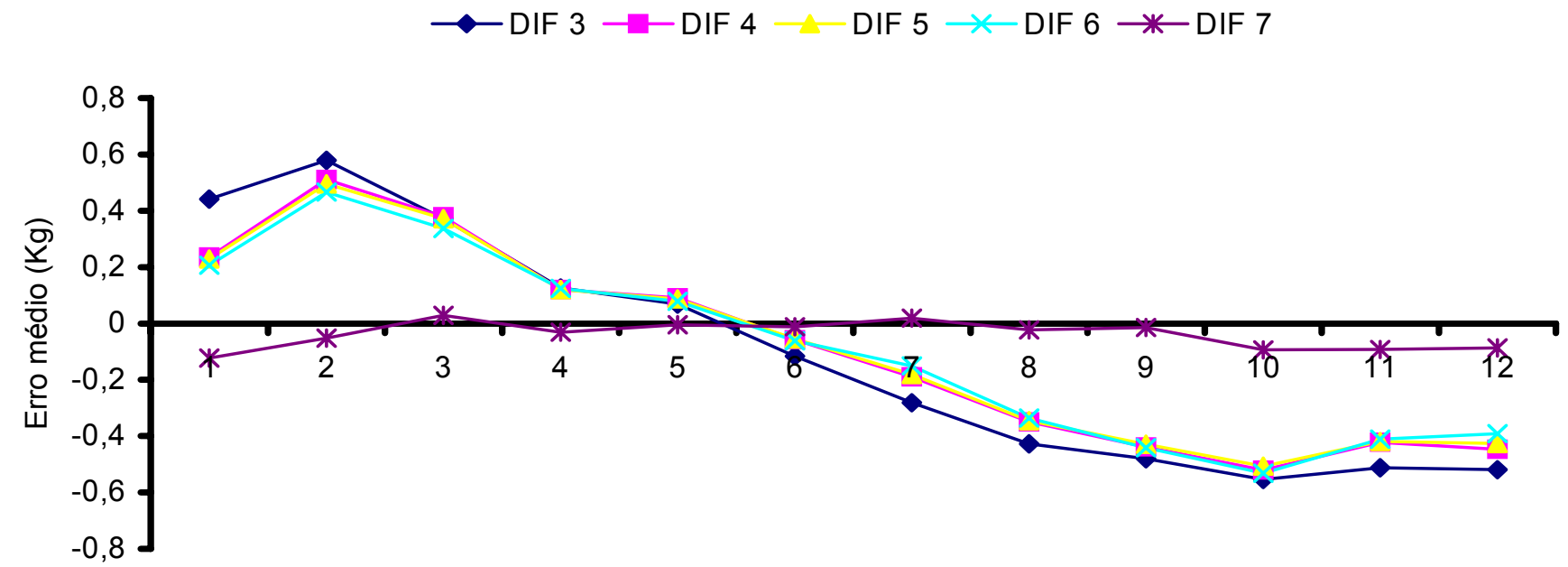

Estádio da lactação em meses

Figura 1. Erro médio da produção diária de leite estimada usando ordenha da manhã.

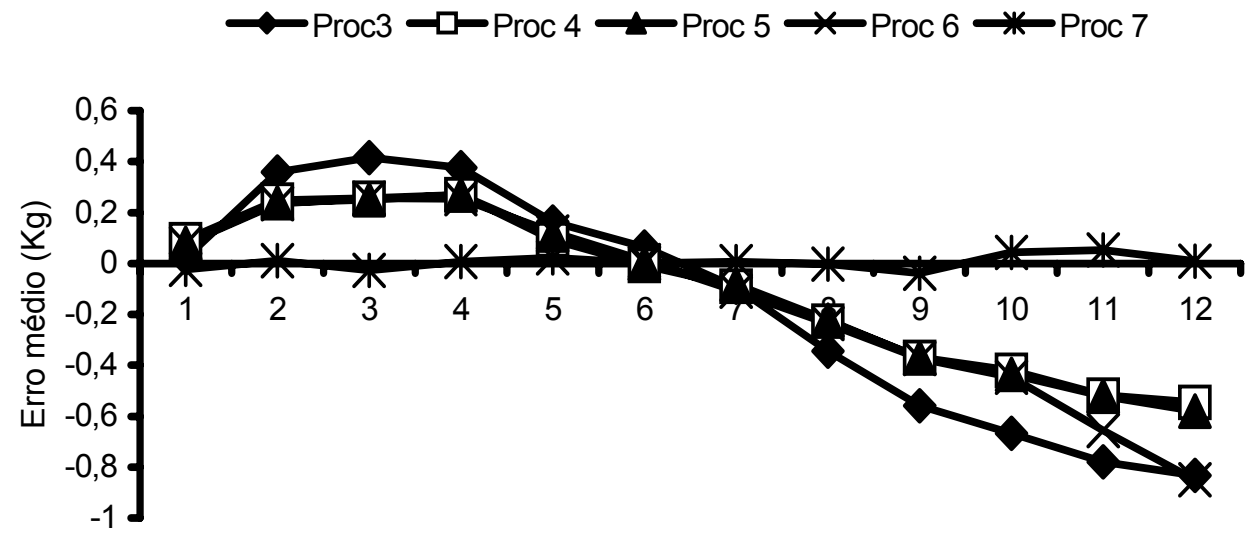

Estádio da lactação em meses

Figura 2. Erro médio da produção diária de leite estimada usando ordenha da tarde.

Tabela 3. Equações de regressão para estimação da produção diária usando ordenha da manhã ou da tarde e diferentes intervalos de ordenha - procedimento 6 


\begin{tabular}{|c|c|}
\hline Intervalo & Equação \\
\hline \multicolumn{2}{|l|}{ Manhã } \\
\hline$<540$ & ----- \\
\hline $540-600$ & ----- \\
\hline $601-660$ & $1.61241+1.76110 * \mathrm{c}_{\mathrm{m}}+0.00786 *(\mathrm{DEL}-158)$ \\
\hline $661-720$ & $1.61546+1.78676 * c_{m}+0.00128 *(D E L-158)$ \\
\hline $721-780$ & $0.33600+1.77178 * c_{m}+0.00387 *(D E L-158)$ \\
\hline $781-810$ & $-0.42968+1.84604 * c_{m}-0.00252 *(D E L-158)$ \\
\hline$>810$ & $0.71044+1.69011 * \mathrm{c}_{\mathrm{m}}-0.00279 *(\mathrm{DEL}-158)$ \\
\hline \multicolumn{2}{|r|}{ 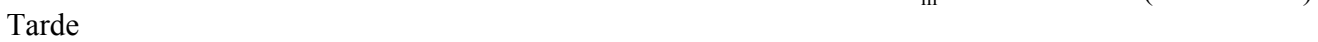 } \\
\hline$<540$ & $7.78246+1.58346 * c_{t}-0.00088 *(D E L-158)$ \\
\hline $540-600$ & $3.71488+1.96210 * c_{t}+0.00185 *(D E L-158)$ \\
\hline $601-660$ & $4.93995+1.79463 * c_{t}-0.00330 *(D E L-158)$ \\
\hline $661-720$ & $2.48420+1.88167 * c_{t}-0.00187 *(D E L-158)$ \\
\hline $721-780$ & $2.09389+1.85560 * c_{t}-0.00002 *(D E L-158)$ \\
\hline $781-810$ & - \\
\hline$>810$ & ----- \\
\hline
\end{tabular}

Como exemplo de aplicação do procedimento 6, considere uma vaca que produziu $23 \mathrm{~kg}$ de leite na ordenha da manhã aos 250 dias de lactação, em um rebanho em que as ordenhas da manhã e a da tarde são realizadas, respectivamente, às $6 \mathrm{e}$ às 14 horas. $\mathrm{O}$ intervalo entre a ordenha da manhã e a da tarde anterior é, portanto, de 960 minutos. A produção diária estimada será $0.71044+(1.69011$ x 23) $-0.00279(250-158)$ $=39,3 \mathrm{~kg}$ de leite.

O resultado da comparação dos sistemas de controle para estimação da produção até 305 dias, para primeira e outras lactações, encontrase na Tab. 4. A produção na primeira lactação foi superestimada e nas outras lactações subestimada usando-se os quatro sistemas. Alternância dos controles da manhã e da tarde, iniciando-se com o controle da manhã foi um pouco mais acurada do que alternância começando com a ordenha da tarde. Semelhante aos resultados de vários autores (Schaeffer e Rennie, 1976; Smith e Pearson, 1981), esses sistemas foram superiores aos sistemas com somente controles da manhã ou somente controles da tarde. As correlações entre produção observada e estimada foram 0,99 e 0,97 , respectivamente, para os sistemas com alternância dos controles e sistemas com todos os controles da manhã ou todos os controles da tarde.

Tabela 4. Médias (kg) dos desvios da produção até 305 dias estimada da observada e correlações entre produção estimada e observada para quatro sistemas de controle

\begin{tabular}{|c|c|c|c|c|c|c|}
\hline \multirow{3}{*}{ Sistema de controle } & \multicolumn{6}{|c|}{ Lactação } \\
\hline & \multicolumn{3}{|c|}{ Primeira } & \multicolumn{3}{|c|}{ Outras } \\
\hline & $\mathrm{n}$ & Média & $\mathrm{r}$ & $\mathrm{n}$ & Média & $\mathrm{r}$ \\
\hline Alternância M-T, primeiro M & 503 & $47 \pm 203$ & 0,99 & 612 & $-39 \pm 245$ & 0,99 \\
\hline Alternância M-T, primeiro T & 506 & $44 \pm 209$ & 0,99 & 615 & $-38 \pm 260$ & 0,99 \\
\hline Todos os controles, $\mathrm{M}$ & 503 & $32 \pm 322$ & 0,97 & 612 & $-19 \pm 388$ & 0,97 \\
\hline Todos os controles, $\mathrm{T}$ & 506 & $58 \pm 352$ & 0,97 & 615 & $-57 \pm 446$ & 0,97 \\
\hline
\end{tabular}

$\mathrm{M}=$ manhã; $\mathrm{T}=$ tarde

\section{CONCLUSÕES}

O procedimento 6 , em que se ajustou uma regressão para cada classe de intervalo de ordenha e se incluíram dias em lactação como co-variável, foi o mais acurado para estimação da produção diária com base na produção das ordenhas da manhã e da tarde. Para se recomendar esse procedimento, seriam, ainda, convenientes estudos dos componentes do leite e CCS que requerem conjuntos específicos de dados. O controle de uma única ordenha, em vez de duas, alternando-se as ordenhas da manhã e da tarde, em meses subseqüentes e iniciando-se 
com o da manhã, foi o melhor sistema para estimação da produção até 305 dias. Entretanto, para que este sistema seja recomendado, é necessário conhecer os efeitos da alternância dos controles da manhã e da tarde sobre valores genéticos de vacas e touros.

\section{REFERÊNCIAS BIBLIOGRÁFICAS}

BRASIL. Portaria n. 45, de 10 de outubro de 1986. Normas técnicas para execução do serviço de controle leiteiro em bovídeos. Diário Oficial [da República Federativa do Brasil], Brasília, DF, n.195, p.15532-15535,15/10/86. Seção I.

CASSANDRO, M.; CARNIER, P.; GALLO, L. et al. Bias and accuracy of single milking testing schemes to estimate daily and lactation milk yield. J. Dairy Sci., v.78, p.2884-2893, 1995.

COSTA, C.N.; TEIXEIRA, N.M.; FREITAS, A.F. et al. Sumário Nacional de Touros da Raça Holandesa - 2003, Juiz de Fora: Embrapa Gado de Leite, 2003, 40p. (Série Documentos, 93).

COSTA, C.N.; TEIXEIRA, N.M.; FREITAS, A.F. et al. Trends in milk recording of the Holstein breed in Brasil. In: ICAR SESSION AND INTERBULL MEETING, 34., 2004, Sousse. Proceedings... Sousse, Tunísia, 2004.

DICKINSON, F.N.; MCDANIEL, B.T. Singlemilking yields versus 24-hour yields for estimating lactation milk production by the Test Interval Method. J. Dairy Sci., v.53, p.200-207, 1970 .

EVERETT, R.W.; WADELL. Source of variation affecting ratio factors for estimating total daily yield from individual milkings. $J$. Dairy Sci., v.53, p.1430-1435, 1970.
LEE, A.J.; WARDROP, J. Predicting daily milk yield, fat percentage and protein percentage from morning and afternoon tests. J. Dairy Sci., v.67, p.351-360, 1984.

MCDANIEL, B.T. Accuracy of sampling procedures for estimating lactation yields: a review. J. Dairy Sci., v.52, p.1742-1761, 1969.

PUTNAM, D.N.; GILMORE, H.C. Alternate AM-PM testing for dairy herd improvement association program- operational procedures. $J$. Dairy Sci., v.52, suppl.1, p.985, 1968. (Abstr.).

PUTNAM, D.N.; GILMORE, H.C. Evaluation on an alternate AM and PM monthly testing plan and its application for use in the Dairy Herd Improvement Association. J. Dairy Sci., v.51, suppl. 1, p.985, 1968. (Abstr.).

SCHAEFFER, L.R.; JAMROZIK, J.; VAN DORP, R. et al. Estimating daily yields of cows from different milking schemes. Liv. Prod. Sci., v.65, p.219-227, 2000.

SCHAEFFER,L.R.; RENNIE， J.C. AM-PM testing for estimating lactation yields. Can. J. Anim. Sci., v.56, p.9-15, 1976.

SMITH, J.W.; PEARSON, R.E. Development and evaluation of alternate testing procedures for official records. J. Dairy Sci., v.64, p.466-474, 1981.

VERNEQUE, R.S.; MARTINEZ, M.L.; TEODORO, R.L. et al. Programa Nacional de Melhoramento do Gir Leiteiro: resultado do teste de progênie - $11^{0}$ Grupo. Juiz de Fora: Embrapa Gado de Leite, 2003. 35p. (Série Documentos, 89).

WIGGANS, G.R. Methods to estimate milk and fat yields from AM/PM plans. J. Dairy Sci., v.64, p.1621-1624, 1981. 